\title{
Non-Associative Property of 123-Avoiding Class of Aunu Permutation Patterns
}

\author{
Aminu Alhaji Ibrahim, Sa'idu Isah Abubakar \\ Department of Mathematics, Sokoto State University, Sokot, Nigeria \\ Email: aminualhaji40@gmail.com,siabubakar82@gmail.com
}

Received 24 November 2015; accepted 24 January 2016; published 28 January 2016

Copyright (C) 2016 by authors and Scientific Research Publishing Inc.

This work is licensed under the Creative Commons Attribution International License (CC BY). http://creativecommons.org/licenses/by/4.0/

c) (i) Open Access

\section{Abstract}

This paper presents the non-associative and non-commutative properties of the 123-avoiding patterns of Aunu permutation patterns. The generating function of the said patterns has been reported earlier by the author [1] [2]. The paper describes how these non-associative and non commutative properties can be established by using the Cayley table on which a binary operation is defined to act on the 123-avoiding and 132-avoiding patterns of Aunu permutations using a pairing scheme. Our results have generated larger matrices from permutations of points of the Aunu patterns of prime cardinality. It follows that the generated symbols can be used in further studies and analysis in cryptography and game theory thereby providing an interdisciplinary approach and applications of these important permutation patterns.

\section{Keywords}

Non-Associative, Non-Commutative, Permutation, Pattern Avoidance, 123-Avoiding, Aunu Patterns, Cayley Tables, Ecetra

\section{Introduction}

Non-associative algebraic structures arise in many situations. Cayley octonions are a notorious example, but there are far more; for example, nonassociative loop arise in cordinatization of projective planes and the Einstein velocity addition in relativity theory also forms a nonassociative loop. Self distributive algebras appear naturally in the study of Braids [3].

The 123-avoiding class of the Aunu permutation patterns which have been found to be of both combinatorics and group theoretic importance [1] can also be used to construct some structures which are non-associative as well as non-commutative using Cayley table with a binary operation defined to act on such patterns.

Non-associative structures include structures like groupoids, quasigroup and loops, nonassociative semi-rings 
as well as self distributive algebras and mediality.

The oldest and most developed discipline of nonassociative algebra originated in 1930s in works of Sushkevich, Moufang, Bol, Mordorch, and others, see [4] for comprehensive historical notes. One of the earliest surveys on nonassociative algebras is the article by [5] which introduced the phrase "rings that are linearly associative". The first book in the English Language devoted to a systematic study of nonassociative algebras is [6]. A collection of open research problems in algebra is the Dniester Notebook [7]; The survey article by [8] is from the same period. Three books on Jordan algebras which contain substantial material on general non associative algebras are [9]-[11].

Recent researches appear in the Proceedings of International Conferences on Nonassociative Algebra and its Applications [12]-[14]. The topic is covered by several books [15]-[19] that study various aspects of the theory, and reflect different eras of non-associative mathematics.

In order to make this paper more self-contained, some notation overview is here under presented of some key concepts used in the paper.

\subsection{Permutation Patterns}

An arrangement of the objects $1,2, \cdots, n$ is a sequence consisting of these objects arranged in any order. When in addition, a particular order of arrangement is desired, such an arrangement becomes an ordered arrangement governed by a pattern $\sigma$ and each such permutation $\sigma \in S_{n}$ naturally results into a certain arrangement of $1,2, \cdots, n$ given by

$$
\sigma(1) \sigma(2) \cdots \sigma(n)
$$

which is called the arrangement associated with a permutation pattern $\sigma$ of points of a nonempty set

$$
\Omega=\{1,2, \cdots, n\}
$$

Given a sequence $\pi$ consisting of $n$ elements arranged in a given pattern and another sequence $\sigma$ having $m$ elements such that $m<n$, then $\sigma$ is said to be contained as a pattern in $\pi$ provided $\pi$ has a subsequence which is order isomorphic to $\sigma$. If $\pi$ does not contain $\sigma$ it is said to avoid it. The set of all the $\sigma$-avoiding permutations is denoted $S_{n}(\sigma)$.

It is useful to differentiate between a subsequence and a subword. For instance, if $\sigma=4132 \in S_{4}$ then $\pi=78364521 \in S_{8}$ contains $\sigma$ as a subword since $\rho(8364)=4132$. However, $\pi=54321 \in S_{5}$ does not contain $\sigma$ as a subword although it does contain it as a subsequence. Occurrences of subwords can be overlapped. As an example the sequence $5716243 \in S_{7}$ contains two occurrences of $\sigma: 7162$ and 6243

Determination of $\left|S_{n}(\sigma)\right|$ has remained a hard and intractable problem for a given $\sigma$ containing more than three elements. This is among the reasons that motivate the author to construct some classes of pattern-avoiding permutations using some special subword (instead of subsequences) governed by some succession schemes [2]. Thus, $A_{n}(132)$ in this context, represents the sub words of length $n \geq 3$ that are (132)-avoiding in $A_{n}$ being the set of strictly consecutive succession scheme containing pairs $i, j$ such that

$$
j=i+1, \quad i, j \in \mathbb{N}
$$

\subsection{Aunu Permutation Patterns}

It was reported by [2] [20] [21] in a generalized and elaborate enumeration scheme of a recursion relation for generation of some special classes of (123) and (132)-avoiding permutation patterns of Aunu patterns. [22] identified a new and more generalized generating function for the Aunu pattern which was based on the method employ by [23]. He also further identified and discussed some other theoretic properties of the Aunu patterns and Aunu Groups especially in relation to integer modulo groups. The theoretical application of both Aunu pattern and Aunu Group from the method of generating function was identified by [22].

\section{Method of Construction}

The basic procedure for generating the special permutation patterns under study, have already been outlined, see for instance [2] [24] as well as [1]. However, for the sake clarity, the basic procedure is once more, highlighted below. 


\section{The Special (123)-Avoiding Scheme}

As a pairing scheme involving pairs of numbers associated by some precedence relation [1] [24]. The governing conditions for the generation of these numbers are outlined below.

The elements are paired in order of precedence

$$
\lambda_{i}, \lambda_{j}, \ni i \Theta j \quad i, j \in C_{n}
$$

where $C_{n}$ is a cycle of length $n$ while $\lambda_{i}, \lambda_{j}$ are in the $i^{\text {th }}$ and $j^{\text {th }}$ positions in the permutation pattern generated by the precedence parameter $\Theta$.

The precedence parameter acts on the elements to produce pairs such as are related as; element and first successor, element and second successor, up to element and $n^{\text {th }}$ successor.

Under the given condition, it is required that the $j^{\text {th }}$ partner shifts in position incrementally corresponding to the $n^{\text {th }}$ succession so that

$$
j=i+1, i+2, \cdots, i+m
$$

where $m \leq n$

The enumeration scheme involves doubt regarding the identity of the first element in the desired pair. Moreover, absolute certainty is desired that by the end of the enumeration, the required pair, whichever it is, is achieved.

We now state an important theorem for the enumeration of these permutation patterns.

\section{Theorem 2.1}

The number of subwords for the permutation patterns under study is enumerated as: $2,3,5,5,8, \cdots$ corresponding to the length (cardinality) of the special (123)-avoiding sequences 5,7,11,13,17, $\cdots[2]$.

\section{Proof}

To prove this let us suppose a permutation $a=(123 \cdots n)$. We now generate a special mapping $\Upsilon$ on $a$ such that elements of the permutation are mapped one on to another governed by a specified order of arrangement as in as in [2] thus:

$$
\begin{aligned}
& a_{1}=1 \rightarrow 2 \rightarrow 3 \rightarrow \cdots \rightarrow n, \\
& a_{2}=1 \rightarrow 3 \rightarrow 5 \rightarrow \cdots \rightarrow(n-1), \\
& \quad \vdots \\
& a_{n}=1 \rightarrow n \rightarrow(n-1) \rightarrow \cdots \rightarrow 2
\end{aligned}
$$

We now rewrite these numbers in the form of sequence as follows:

$$
123 \cdots n, 135 \cdots(n-1) \text { up to } 1 n(n-1) \cdots 2
$$

\section{Results}

We now define a mapping $\Theta: \Omega=\{1,2, \cdots, n\}$ of 132-avoiding patterns and 123-avoiding patterns of Aunu numbers of cardinality $n$ where $n$ is necessarily a prime.

$\Theta: \Omega=\{1,2, \cdots, n\} \rightarrow A_{n(123)}$ where $A_{n(123)}$ represents the (123)-avoiding patterns of Aunu permutation reported in theorem 2.1; and $n$ is prime greater than or equal to five.

Then, for $\Omega=\{1,2,3,4,5\}$

$$
\Theta(123 \text {-Avoiding })=\Theta_{j}^{n}(i)=\left(i+j^{\text {th }} \text { permutation }\right) \text { Modulon. }
$$

where $i$ enumerates the cycles formed in permutations of elements of $\Omega$ and $j$ represents the shift in permutation of elements of the symbols (elements) of $\Omega$ in a pairing scheme defined by (4)

An illustrative Example is provided thus: for $n=5$, a permutation can be generated for $\Omega=\{1,2,3,4,5\}$ using $i$ as first symbol of $\Omega$ in a pairing scheme defined in (6). The first set of permuted elements of $\Omega$ can now be generated by the arrangement of elements of $\Omega$ as pairs of symbols both of increasing magnitude modulo 5; that is, for the first cycle C1 with I = 1 we have: 


$$
\Theta_{j}^{n}(1)=1+0=1, \Theta_{j}^{n}(2)=2+1=3, \Theta_{j}^{n}(3)=3+2=5, \quad \Theta_{j}^{n}(4)=(4+3) \bmod 5=2,
$$

$\Theta_{j}^{n}(5)=(5+4) \bmod 5=4$ completing the first cycle.

without loss of generality, subsequent cycles can be constructed using similar procedure by rearrangement of elements of $\Omega$.

It follows that $\Theta$ provides a shift among points of $\Omega$ corresponding to the length of the non empty set $\Omega$ in a pairing scheme.

The following Tables 1-5 provide summarized results for the aforementioned procedure on $A_{n(123)}$ and for $n=5,7,11,13$ and 17 .

We now use the entries of the Cayley table to test non-associativity of points in Aunu permutations patterns of $5 \leq n \leq 17$.

It can be seen from the above Cayley table that, it is closed under $\Theta$ but non-associative and commutative lawsdo not also hold.

i.e. $1 \Theta(2 \Theta 3) \neq(1 \Theta 2) \Theta 3$ (non-associative)

Also, $(2 \Theta 3) \neq(3 \Theta 2)$ (non-commutative)

It can be shown from Table 2 that the structure is non-associative and non commutative.

Table 1. Cayley table for $n=5$ showing generated points of $\Omega$ as permutations of (132) and (123)-avoiding patterns of Aunu scheme under the action of $\Theta$.

\begin{tabular}{llllll}
\hline$\Theta$ & 1 & 2 & 3 & 4 & 5 \\
1 & 1 & 3 & 5 & 2 & 4 \\
2 & 1 & 4 & 2 & 5 & 3 \\
3 & 1 & 5 & 4 & 3 & 2 \\
\hline
\end{tabular}

Table 2. Cayley table for $n=7$ showing generated points of $\Omega$ as permutations of (132) and (123)-avoiding patterns of Aunu scheme under the action of $\Theta$.

\begin{tabular}{llllllll}
\hline$\Theta$ & 1 & 3 & 4 & 5 & 6 & 7 \\
1 & 1 & 3 & 5 & 7 & 2 & 4 & 6 \\
2 & 1 & 4 & 7 & 3 & 6 & 2 & 5 \\
3 & 1 & 5 & 2 & 6 & 3 & 7 & 4 \\
4 & 1 & 6 & 4 & 2 & 7 & 5 & 3 \\
5 & 1 & 7 & 6 & 5 & 4 & 3 & 2 \\
\hline
\end{tabular}

Table 3. Cayley table for $n=11$ showing generated points of $\Omega$ as permutations of (132) and (123)-avoiding patterns of Aunu scheme under the action of $\Theta$.

\begin{tabular}{cccccccccccccc}
$\Theta$ & 1 & 2 & 3 & 4 & 5 & 6 & 7 & 8 & 9 & 10 & 11 \\
\hline 1 & 1 & 3 & 5 & 7 & 9 & 11 & 2 & 4 & 6 & 8 & 10 \\
2 & 1 & 4 & 7 & 10 & 2 & 5 & 8 & 11 & 3 & 6 & 9 \\
3 & 1 & 5 & 9 & 2 & 6 & 10 & 3 & 7 & 11 & 4 & 8 \\
4 & 1 & 6 & 11 & 5 & 10 & 4 & 9 & 3 & 8 & 2 & 7 \\
5 & 1 & 7 & 2 & 8 & 3 & 9 & 4 & 10 & 5 & 11 & 6 \\
6 & 1 & 8 & 4 & 11 & 7 & 3 & 10 & 6 & 2 & 9 & 5 \\
7 & 1 & 9 & 6 & 3 & 11 & 8 & 5 & 2 & 10 & 7 & 4 \\
8 & 1 & 10 & 8 & 6 & 4 & 2 & 11 & 9 & 7 & 5 & 3 \\
9 & 1 & 11 & 10 & 9 & 8 & 7 & 6 & 5 & 4 & 3 & 2 \\
\hline
\end{tabular}


Table 4. Cayley table for $n=13$ showing generated points of $\Omega$ as permutations of (132) and (123)-avoiding patterns of Aunu scheme under the action of $\Theta$.

\begin{tabular}{cccccccccccccc}
$\Theta$ & 1 & 2 & 3 & 4 & 5 & 6 & 7 & 8 & 9 & 10 & 11 & 12 & 13 \\
\hline 1 & 1 & 3 & 5 & 7 & 9 & 11 & 13 & 2 & 4 & 6 & 8 & 10 & 12 \\
2 & 1 & 4 & 7 & 10 & 13 & 3 & 6 & 9 & 12 & 2 & 5 & 8 & 11 \\
3 & 1 & 5 & 9 & 13 & 4 & 8 & 12 & 3 & 7 & 11 & 2 & 6 & 10 \\
4 & 1 & 6 & 11 & 3 & 8 & 13 & 5 & 10 & 2 & 7 & 12 & 4 & 9 \\
5 & 1 & 7 & 13 & 6 & 12 & 5 & 11 & 4 & 10 & 3 & 9 & 2 & 8 \\
6 & 1 & 8 & 2 & 9 & 3 & 10 & 4 & 11 & 5 & 12 & 6 & 13 & 7 \\
7 & 1 & 9 & 4 & 12 & 7 & 2 & 10 & 5 & 13 & 8 & 3 & 11 & 6 \\
8 & 1 & 10 & 6 & 2 & 11 & 7 & 3 & 12 & 8 & 4 & 13 & 9 & 5 \\
9 & 1 & 11 & 8 & 5 & 2 & 12 & 9 & 6 & 3 & 13 & 10 & 7 & 4 \\
10 & 1 & 12 & 10 & 8 & 6 & 4 & 2 & 13 & 11 & 9 & 7 & 5 & 3 \\
11 & 1 & 13 & 12 & 11 & 10 & 9 & 8 & 7 & 6 & 5 & 4 & 3 & 2 \\
\hline
\end{tabular}

Table 5. Cayley table for $n=17$ showing generated points of $\Omega$ as permutations of (132) and (123)-avoiding patterns of Aunu scheme under the action of $\Theta$.

\begin{tabular}{cccccccccccccccccc}
\hline$\Theta$ & 1 & 2 & 3 & 4 & 5 & 6 & 7 & 8 & 9 & 10 & 11 & 12 & 13 & 14 & 15 & 16 & 17 \\
\hline 1 & 1 & 3 & 5 & 7 & 9 & 11 & 13 & 15 & 17 & 2 & 4 & 6 & 8 & 10 & 12 & 14 & 16 \\
2 & 1 & 4 & 7 & 10 & 13 & 16 & 2 & 5 & 8 & 11 & 14 & 17 & 3 & 6 & 9 & 12 & 15 \\
3 & 1 & 5 & 9 & 13 & 17 & 4 & 8 & 12 & 16 & 3 & 7 & 11 & 15 & 2 & 6 & 10 & 14 \\
4 & 1 & 6 & 11 & 16 & 4 & 9 & 14 & 2 & 7 & 12 & 17 & 5 & 10 & 15 & 3 & 8 & 13 \\
5 & 1 & 7 & 13 & 2 & 8 & 14 & 3 & 9 & 15 & 4 & 10 & 16 & 5 & 11 & 17 & 6 & 12 \\
6 & 1 & 8 & 15 & 5 & 12 & 2 & 9 & 16 & 6 & 13 & 3 & 10 & 17 & 7 & 14 & 4 & 11 \\
7 & 1 & 9 & 17 & 8 & 16 & 7 & 15 & 6 & 14 & 5 & 13 & 4 & 12 & 3 & 11 & 2 & 10 \\
8 & 1 & 10 & 2 & 11 & 3 & 12 & 4 & 13 & 5 & 14 & 6 & 15 & 7 & 16 & 8 & 17 & 9 \\
9 & 1 & 11 & 4 & 14 & 7 & 17 & 10 & 3 & 13 & 6 & 16 & 9 & 2 & 12 & 5 & 15 & 8 \\
10 & 1 & 12 & 6 & 17 & 11 & 5 & 16 & 10 & 4 & 15 & 9 & 3 & 14 & 8 & 2 & 13 & 7 \\
11 & 1 & 13 & 8 & 3 & 15 & 10 & 5 & 17 & 12 & 7 & 2 & 14 & 9 & 4 & 16 & 11 & 6 \\
12 & 1 & 14 & 10 & 6 & 2 & 15 & 11 & 7 & 3 & 16 & 12 & 8 & 4 & 17 & 13 & 9 & 5 \\
13 & 1 & 15 & 12 & 9 & 6 & 3 & 17 & 14 & 11 & 8 & 5 & 2 & 16 & 13 & 10 & 7 & 4 \\
14 & 1 & 16 & 14 & 12 & 10 & 8 & 6 & 4 & 2 & 17 & 15 & 13 & 11 & 9 & 7 & 5 & 3 \\
15 & 1 & 17 & 16 & 15 & 14 & 13 & 12 & 11 & 10 & 9 & 8 & 7 & 6 & 5 & 4 & 3 & 2 \\
\hline
\end{tabular}

E.g. $2 \Theta(4 \Theta 5) \neq(2 \Theta 4) \Theta 5$

$1 \Theta(2 \Theta 3) \neq(1 \Theta 2) \Theta 3$

$3 \Theta(4 \Theta 5) \neq(3 \Theta 4) \Theta 5$

Also, $4 \Theta 5 \neq 5 \Theta 4,2 \Theta 3 \neq 3 \Theta 2$, etc.

It can be seen from Table 3 that:

$7 \Theta(8 \Theta 9) \neq(7 \Theta 8) \Theta 9$

$3 \Theta(5 \Theta 7) \neq(3 \Theta 5) \Theta 7$

$5 \Theta(6 \Theta 7) \neq(5 \Theta 6) \Theta 7$ etc.

Also, $8 \Theta 9 \neq 9 \Theta 8$,

It can also be shown from Table 4 that associativity and commutivity with respect to binary operation $\Theta$ do 
not hold.

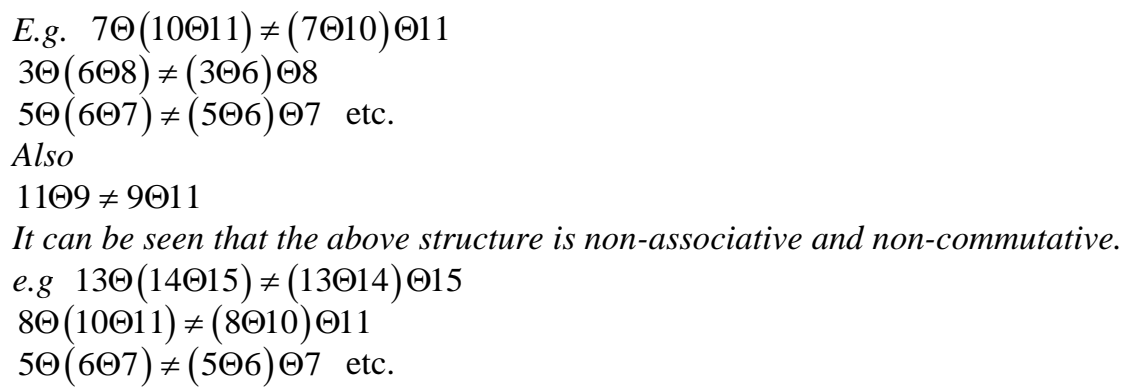

Also, $14 \Theta 15 \neq 15 \Theta 14$, 5 , etc.

This can be stated in a general form as, taken any $x, y, z$ from 132-avoiding patterns and the 123-avoiding class of Aunu permutations patterns with a binary operation $\Theta$ defined, $x \Theta(y \Theta z) \neq(x \Theta y) \Theta z$. Likewise $x \Theta y \neq y \Theta x$. This has clearly shown to us that, the above constructed structures using Cayley table are non-associative and non-commutative.

\section{Conclusion}

It follows that the permuted structures of Aunu scheme give rise to non-associative structures where points in Aunu permutations are regarded as elements of the derived sets in relation to pairing scheme modulo $n$, where $n$ is necessarily a prime $\geq 5$. The precedence/pairing parameter $\Theta$ generates some sequences which can be studied further in relation to group action and transformation schemes. Further algebraic properties can also be investigated on these structures by using graph theoretic methods. Further, the matrices obtained from the resulting Cayley table appear to possess some combinatorial and number theoretic properties which can be investigated in subsequent researches.

\section{References}

[1] Ibrahim, A.A. (2005) On the Combination of Succession in It-5 Element Sample. Abacus Journal of Mathematics Association on Nigeria, 32, 410-415.

[2] Ibrahim, A.A. and Audu, M.S (2005) Some Group Theoretic Properties of Certain Class of (123) and (132) Avoiding Patterns of Certain Numbers: An Enumeration Scheme. African Journal of Natural Science, 8, 79-84.

[3] Dehornoy, P. (2000) Braids and Self-Distributivity. Progress in Mathematics, Vol. 192. Birkhauser, Besel.

[4] Pflugfelder, H.O. (2000) Historical Notes on Loop Theory. Commentationes Mathematicae Universitatis Carolinae, 41, 359-370.

[5] Shirshov, A.I. (1958) Some Problems in the Theory of Rings that Are Nearly Associative. Uspekhi Matematicheskikh Nauk, 13, 3-20.

[6] Schefer, R.D. (1966) An Introduction to Nonassociative Algebras. Dover Publication, New York.

[7] Filippov, V.T., Kharchenko, V.K. and Shestakov, I.P., Eds. (1993) The Dniester Notebook: Unsolved Problems in the Theory of Rings and Modules. 4th Edition, Springer-Verlag, New York.

[8] Kuzmin, E.N. and Shestakov, I.P. (1995) Nonassociative Structures. In: Algebra VI, Encyclopaedia of Mathematical Sciences 57, Springer Verlag, Berlin, 197-280.

[9] Braun, H. and Koecher, M. (1966) Jordan Algebren (German). Springer-Verlag, Berlin and New York. http://dx.doi.org/10.1007/978-3-642-94947-0

[10] Jacobson, N. (1968) Structure and Representations of Jordan Algebras. American Mathematical Society, Providence.

[11] McCrimmon, K. (2004) A Taste of Jordan Algebras. Springer-Verleg, New York.

[12] Gonzalez, S., Ed. (1993) Proceedings of the 3rd International Conference on Non-Associative Algebra and Its Applications, Oviedo, 12-17 July 1993, 400-410.

[13] Costa, R., Grishkov, A., Guzzo Jr., H. and Peresi, L.A., Eds. (1998) Proceedings of the 4th International Conference on Non-Associative Algebra and Its Applications, Sao Paulo, 19-25 July 1998, 34-37.

[14] Sabinin, L., Sbitneva, L., and Shestakov, I.P., Eds. (2003) Proceedings of the 5th International Conference on Non-Associative Algebra and Its Applications, Oaxtepec, 27th July-2 August 2003, 44-45.

[15] Bruck, R.H. (1958) A Survey of Binary System. Springer, Berlin. 
[16] Belousov, V.D. (1967) Osnovyteoiikvazigrupp I lup. Nauka, Moskva. (In Russian)

[17] Pflugfelder, H.O. (1990) Quasigroups and Loops: Introduction. Herdermann, Berlin.

[18] Chein, O., Pfulgfelder, H.O. and Smith, J.D.H., Eds. (1990) Quasigroups and loops: Theory and Applications. Heldermann, Berlin.

[19] Nagy, P. and Strambach, K. (2002) Loops in Group Theory and Lie Theory. de Gruyter, Berlin. http://dx.doi.org/10.1515/9783110900583

[20] Ibrahim, A.A. and Audu, M.S. (2007) An Wreath Product of Permutation Graphs Proyessiones. Journal Mathematics Autotgasta, 26, 73-90.

[21] Ibrahim, A.A. and Audu, M.S. (2010) Some Group Theoretic Properties of Certain Class of (123) and (132) Avoiding Patterns of Numbers: An Enumeration Scheme. African Journal of National Sciences, 8, 79-84.

[22] Usman, A. and Ibrahim, A.A. (2011) A New Generating Function for Aunu Patterns; Application in Integer Group Modulon. Nigeria Journal of Basic and Applied Sciences, 19, 1-4.

[23] Ibrahim, A.A. (2006) Some Graph Theoretical Properties of (132)—Avoiding Patterns of Certain Class. Nigerian Journal of Renewable Energy, 14, 21-24.

[24] Ibrahim, A.A. (2004) On Wreath Product of Permutation Groups and Algebraic Theoretic Properties of Bara’at AlDhimmah Models. PhD Thesis, Usmanu Danfodiyo University, Sokoto. 